\title{
CONSERVAÇÃO DE GOIABAS TRATADAS COM EMULSÕES DE CERA DE CARNAÚBA ${ }^{1}$
}

\author{
ANGELO PEDRO JACOMINO ${ }^{2,5^{*}}$; RAMÓN MARTÍNEZ OJEDA ${ }^{3}$; RICARDO ALFREDO KLUGE ${ }^{4,5}$; JOÃO ALEXIO \\ SCARPARE FILHO ${ }^{2,5}$
}

\begin{abstract}
RESUMO - A goiaba é um fruto muito perecível. Assim, objetivou-se avaliar os efeitos de ceras à base de carnaúba na conservação pós-colheita de goiabas Pedro Sato sob condição ambiente. Utilizaram-se cinco ceras comerciais: Citrosol AK (18\%), Citrosol M (10\%), Fruit wax (18 a $21 \%)$, Meghwax ECF-100 (30\%) e Cleantex wax (18,5 a 20,5\%), as quais foram aplicadas manualmente, na proporção de 0,15 a $0,20 \mathrm{~mL}$ por fruta. Frutas sem aplicação de cera foram utilizadas como controle. O delineamento experimental foi inteiramente casualizado com 6 tratamentos, 4 repetições e 5 frutas por parcela. As goiabas foram caracterizadas imediatamente após a colheita e avaliadas aos 2, 4 e 6 dias após a aplicação dos tratamentos. As ceras exerceram pouca influência nos teores de sólidos solúveis totais, acidez total titulável e ácido ascórbico, porém, foram eficientes em retardar o amadurecimento, reduzir a perda de massa e a incidência de podridões. A cera Meghwax ECF-100 apresenta potencial para utilização em goiabas, porém há necessidade de ser avaliada em maior diluição, para evitar alterações indesejáveis.
\end{abstract}

Palavras-chave: Psidium guajava, armazenamento, atmosfera modificada

\section{POSTHARVEST CONSERVATION OF GUAVAS THROUGH CARNAUBA WAX EMULSION APPLICATIONS}

ABSTRACT - Guavas are very perishable fruits. Therefore, the objective of the present work was to evaluate the effects of several carnauba based waxes in the postharvest life of Pedro Sato guavas under room conditions. Five commercial waxes were used: Citrosol AK (18\%), Citrosol M (10\%), Fruit wax (18 a 21\%), Meghwax ECF-100 (30\%) e Cleantex wax (18,5 a 20,5\%). The waxes were applied manualy in the rate of 0.15 to $0.20 \mathrm{~mL}$ of wax per fruit. Control fruits were not treated. The experiment was conducted in a completely randomized design with 6 treatments, 4 replicates per treatment and 5 fruits as experimental unit. Guavas were evaluated at harvest and at every 2 days until the $6^{\text {th }}$ day after treatments. Waxing had little effect on total soluble solids, total titratable acidity and ascorbic acid contents. However, the waxes were efficient in delaying ripening and reducing mass loss and decay incidence. The Meghwax ECF-100 shows potential for its use in guavas, although it needs to be tested in lower concentrations to avoid ripening problems.

Key words: Psidium guajava, storage, modified atmosphere.

\section{INTRODUÇÃO}

A goiaba (Psidium guajava L.) é um fruto muito perecível, com curto período de conservação em temperatura ambiente, o que obriga a uma comercialização rápida para evitar perdas. Os principais aspectos de deterioração são o rápido amolecimento, a perda de coloração verde e do brilho e a incidência de podridões (Jacomino, 1999).

Vários métodos podem ser empregados para ampliar a vida de prateleira das frutas em geral. Estes métodos incluem o uso de atmosfera modificada, que pode ser pelo acondicionamento das frutas em filmes plástico ou pelo recobrimento com ceras especiais. Estes tratamentos modificam o ar circundante e interno da fruta, reduzindo os níveis de $\mathrm{O}_{2}$ e aumentando os níveis de $\mathrm{CO}_{2}$. Conseqüentemente, reduzem o metabolismo do vegetal, retardando a senescência (Chitarra \& Chitarra, 1990; Gorris \& Peppelenbos, 1992). Em goiabas têm sido utilizadas embalagens plásticas individuais associadas ao vácuo parcial, especialmente nos frutos destinados à exportação. Porém, há uma série de problemas relativos à alteração de sabor e custos da embalagem e da operação. As ceras não são usadas comercialmente, mas podem se constituir numa alternativa viável.

A cera à base de carnaúba vem sendo testada em frutas e hortaliças. Obtida a partir de uma palmeira brasileira, tem sido comercializada sob inúmeras marcas, em diferentes concentrações e misturas. Pode ser aplicada em produtos dos quais também se consome a casca, devido ao fato de não ser tóxica. Confere brilho e reduz a perda de matéria fresca dos produtos, além de ser facilmente removível com água, se necessário (Hagenmaier \& Baker, 1994).

Singh \& Chauhan (1982), aplicando ceras de carnaúba em goiabas Sardar, verificaram eficiência na redução da perda de massa dos frutos. Brown \& Wills (1983), além de encontrarem redução na perda de massa, também determinaram um aumento no brilho da casca e nenhuma alteração na coloração da casca, na taxa de respiração e na produção de etileno. Singh et al. (1984) verificaram que o uso de ceras de carnaúba em goiabas Allahabad Safeda aumentou em 3 dias a vida útil das mesmas, com manutenção da firmeza. Pivetta et al. (1992) e Tavares (1993) obtiveram aumento na conservação de goiabas Rica e Paluma, pela aplicação de cera Sta Fresh, também à base de carnaúba. McGuire \& Hallman (1995) verificaram significativa redução na perda da firmeza em goiabas tratadas com cera à base de carnaúba e McGuire (1997) observou retardo de dois dias no amadurecimento de goiabas pelo tratamento térmico associado à cera de carnaúba.

A película de cera aplicada na superfície do produto vegetal apresenta diferentes taxas de permeabilidade ao $\mathrm{O}_{2}, \mathrm{CO}_{2}$ e ao vapor d'água em função das propriedades da matéria prima, de sua concentração e da espessura da película. A combinação adequada destes fatores é variável para cada fruta, conforme suas características fisiológicas (Amarante et al., 2001).

Considerando o curto período de conservação pós-colheita de goiabas em condição ambiente, objetivou-se no presente trabalho avaliar o efeito de ceras à base de carnaúba na conservação de goiabas Pedro Sato visando o aumento de sua vida útil após a colheita.

\section{MATERIALE MÉTODOS}

Goiabas Pedro Sato cultivadas em pomar comercial no município de Vista Alegre do Alto - SP, situado a $48^{\circ} 21^{\prime}$ (W) e $21^{\circ} 10^{\prime}$ (S), foram colhidas no início da manhã, selecionadas e imediatamente transportadas ao Laboratório de Pós-Colheita do Departamento de Produção Vegetal da USP/ESALQ, em Piracicaba - SP. Foram utilizadas frutas sem defeitos, no estádio de maturidade fisiológica e com massa de $168 \pm 15 \mathrm{~g}$.

As goiabas foram padronizadas por parcelas e submetidas à imersão em solução de hipoclorito de sódio (150ppm), colocadas para secar em ambiente ventilado e submetidas à aplicação de emulsões comerciais de cera de carnaúba nas seguintes concentrações: Citrosol AK $=18 \%$; Citrosol M = 10\%; Fruit wax = 18 a 21\%; Meghwax ECF-100 = $30 \%$ e Cleantex wax $=18,5$ a 20,5\%. Frutas sem aplicação de cera foram

\footnotetext{
${ }^{1}$ (Trabalho 008/2003). Recebido: 28/12/2002. Aceito para publicação: 28/08/2003.

2 Prof. Dr., Depto. de Produção Vegetal, USP/ESALQ, C.P. 9, CEP 13418-900 - Piracicaba, SP.

${ }^{3}$ Pós-Graduando em Fitotecnia, Depto. Produção Vegetal, USP/ESALQ, CP 09, CEP 13418-900 - Piracicaba, SP

${ }^{4}$ Prof. Dr., Depto. de Ciências Biológicas, USP/ESALQ, C.P. 9, CEP 13418-900 - Piracicaba, SP.

${ }^{5}$ Bolsista do CNPq.

*Autor correspondente <jacomino@esalq.usp.br>
} 
utilizadas como controle.

A aplicação das emulsões de cera foi manual, de forma a cobrir toda a superfície das frutas com uma fina camada. Com o auxílio de uma pipeta graduada de $1 \mathrm{~mL}$ foi colocado 0,15 a $0,20 \mathrm{~mL}$ de cera em cada fruta e com a mão espalhou-se a cera uniformemente sobre a fruta. Esta quantidade aplicada corresponde a aproximadamente um litro de cera por tonelada de fruta. Posteriormente as frutas foram armazenadas sobre bancadas, em condição ambiente $\left(25 \pm 2^{\circ} \mathrm{C}\right.$ e $\left.75 \pm 5 \% \mathrm{UR}\right)$.

O delineamento experimental foi inteiramente casualizado com 6 tratamentos e 4 repetições de 5 frutas por parcela, com 4 épocas de avaliação.

As frutas foram caracterizadas imediatamente após a colheita e avaliadas após 2, 4 e 6 dias de conservação.

As variáveis analisadas foram: a) Perda de massa: determinada pela diferença entre a massa inicial e a massa final com balança digital e expressa em porcentagem da massa inicial; b) Teor de sólidos solúveis totais (SST): utilizando-se uma amostra centrifugada da polpa da fruta e determinado através de leitura direta em refratômetro digital, expressando-se os resultados em ${ }^{\circ}$ Brix; c) Teor de ácido ascórbico: determinado por titulação com 2,6 diclorofenilindofenol (DCFI), de acordo com a metodologia de Carvalho et al. (1990); d) Coloração da casca e da polpa: determinada com colorímetro Minolta CR-300 e expressa em L* (Luminosidade), $\mathrm{C}^{*}$ (Chroma), $\mathrm{h}^{\circ}$ (Ângulo de cor). Realizaram-se duas leituras por fruta, em lados opostos da região equatorial para a cor da casca e uma leitura na região central da fruta (placenta), cortada em duas metades para a cor da polpa; e) Firmeza da polpa: determinada com penetrômetro manual com ponteira de $8 \mathrm{~mm}$ de diâmetro, tomando-se duas leituras por fruta, em lados opostos da região equatorial; f) Acidez total titulável (ATT): determinada por titulometria, de acordo com a metodologia descrita por Carvalho et al. (1990); g) Análise sensorial: as goiabas foram cortadas em fatias e oferecidas a 4 provadores treinados que atribuíram notas para sabor e odor estranhos com base em uma escala de 5 pontos, onde: $1=$ ausente, $2=$ leve, $3=$ moderado, $4=$ intenso e $5=$ muito intenso; h) Incidência de podridão: determinada pela contagem do número de frutos com pelo menos uma lesão de diâmetro maior ou igual a $3 \mathrm{~mm}$, sendo os resultados expressos em porcentagem de frutos com podridão.

Os dados coletados foram submetidos à análise de variância (teste F) e as médias foram comparadas pelo teste de Tukey a $5 \%$.

\section{RESULTADOS E DISCUSSÃO}

De maneira geral, houve decréscimo da firmeza ao longo do armazenamento (Tabela 1). As frutas foram colhidas com firmeza da polpa de $47,74 \mathrm{~N}$ e após 6 dias de armazenamento os valores encontravamse entre 11,21 e $22,13 \mathrm{~N}$, dependendo do tratamento. Apenas no $6^{\circ}$ dia houve influência da cera, sendo que as frutas tratadas com a cera Meghwax ECF-100 estavam mais firmes que aquelas tratadas com as demais ceras ou não tratadas. Verificou-se também, que as frutas tratadas com a cera Meghwax ECF-100 perderam cerca de 53\% da sua firmeza inicial, enquanto aquelas tratadas com Fruit wax perderam cerca de $64 \%$ e nos demais tratamentos a perda da firmeza variou de 70 a $75 \%$.

$\mathrm{O}$ ângulo de cor $\left(\mathrm{h}^{\circ}\right)$ é uma medida utilizada para expressar a variação da coloração em produtos vegetais (McGuire, 1992). O h ${ }^{\circ}$ assume valor zero para a cor vermelha e $90^{\circ}$ para a cor amarela. Na Tabela 2 observa-se que a cor da casca evoluiu de verde-claro para verde-amarelado, ou seja, o $h^{\circ}$ era de $117,38^{\circ}$ no momento da colheita e diminuiu para valores entre $105,54^{\circ}$ e $110,24^{\circ}$, após 6 dias. De maneira geral, as ceras com maior concentração de soluto (Meghwax ECF-100, Citrossol AK, Fruitwax e Cleantex wax) proporcionaram leve retenção da cor verde da casca em relação às frutas não tratadas ou tratadas com Citrossol M, que possui apenas $10 \%$ de cera de carnaúba. Porém, a cera Meghwax foi a única que diferiu significativamente do controle no $6^{\circ}$ dia de armazenamento, retardando em 2 dias, aproximadamente, a evolução da cor da casca. Esses resultados são semelhantes aos obtidos por Nascimento et al. (1991a, b), Pivetta et al. (1992) e McGuire (1997).
TABELA 1 - Firmeza da polpa de goiabas Pedro Sato submetidas à aplicação de emulsões de cera de carnaúba e armazenadas a $25^{\circ} \mathrm{C}^{1}$.

\begin{tabular}{|c|c|c|c|}
\hline \multirow{2}{*}{ Tratamentos } & \multicolumn{3}{|c|}{ Período de armazenamento (dias) ${ }^{2}$} \\
\hline & 2 & 4 & 6 \\
\hline & & ewton ( & ---------- \\
\hline Citrosol AK & 31,87 & 22,75 & $12,48 \mathrm{~b}$ \\
\hline Fruit wax & 35,20 & 16,88 & $17,28 \mathrm{ab}$ \\
\hline Citrosol M & 32,55 & 17,41 & $13,82 \mathrm{~b}$ \\
\hline Meghwax ECF-100 & 38,32 & 22,82 & $22,13 \mathrm{a}$ \\
\hline Controle & 37,01 & 16,89 & $11,21 \mathrm{~b}$ \\
\hline Cleantex wax & 38,58 & 15,96 & $14,26 \mathrm{~b}$ \\
\hline Médias & 35,58 & 18,78 & 15,19 \\
\hline Teste F (tratamento) & n.s. & n.s. & ** \\
\hline C. V. $(\%)$ & 11,27 & 17,73 & 19,10 \\
\hline
\end{tabular}

${ }^{1}$ Médias seguidas da mesma letra na coluna não diferem pelo teste de Tukey a

$5 \%$; ** e n.s. = significativo a $\mathrm{P} \leq 0,01$ e não significativo, respectivamente.

${ }^{2}$ Firmeza das frutas recém colhidas: $47,74 \mathrm{~N}$.

TABELA 2 - Cor da casca de goiabas Pedro Sato submetidas à aplicação de emulsões de cera de carnaúba e armazenadas a $25^{\circ} \mathrm{C}^{1}$.

\begin{tabular}{lccc}
\hline \multirow{2}{*}{ Tratamento } & \multicolumn{3}{c}{ Período de armazenamento (dias) } \\
\cline { 2 - 4 } & $\mathbf{2}$ & $\mathbf{4}$ & $\mathbf{6}$ \\
\hline & ------- & Angulo de cor $\left.\mathbf{~ ( h}^{\mathbf{0}}\right)^{2}$ & - \\
Citrosol AK & 113,31 & $112,46 \mathrm{ab}$ & $108,59 \mathrm{ab}$ \\
Fruit wax & 113,83 & $113,81 \mathrm{a}$ & $107,75 \mathrm{ab}$ \\
Citrosol M & 111,38 & $110,41 \mathrm{ab}$ & $105,24 \mathrm{~b}$ \\
Meghwax ECF-100 & 112,59 & $113,19 \mathrm{a}$ & $110,24 \mathrm{a}$ \\
Controle & 110,99 & $109,18 \mathrm{~b}$ & $105,54 \mathrm{~b}$ \\
Cleantex wax & 113,32 & $113,22 \mathrm{a}$ & $105,59 \mathrm{ab}$ \\
\hline Médias & 112,57 & 112,04 & 107,32 \\
Teste F (tratamento) & n.s. & $* *$ & $*$ \\
C. V. (\%) & 1,20 & 1,41 & 1,93 \\
\hline
\end{tabular}

Médias seguidas da mesma letra na coluna não diferem pelo teste de Tukey a $5 \%$; ** $*$ e n.s. $=$ significativo a $\mathrm{P} \leq 0,01, \mathrm{P} \leq 0,05$ e não significativo, respectivamente.

${ }^{2} \mathrm{~h}^{\mathrm{o}}$ das frutas recém colhidas $=117,38^{\circ}$.

As mudanças na coloração da casca durante o amadurecimento são devido tanto a processos degradativos quanto a processos sintéticos que ocorrem ao mesmo tempo (Chitarra \& Chitarra, 1990). Durante a conservação das goiabas não foi observado anormalidade no desenvolvimento da coloração.

Quanto à cor da polpa, observou-se que esta mudou de rosa para vermelho com o decorrer do armazenamento (Tabela 3). A cromaticidade indica a saturação ou intensidade da cor. Observou-se que a saturação da cor vermelha aumentou com o armazenamento, passando de rosa $(32,62)$, no início do experimento, para vermelho intenso $(43,73)$, no $6^{\circ}$ dia de armazenamento. As frutas tratadas com Meghwax ECF-100 não desenvolveram a coloração característica desta variedade, diferindo dos demais tratamentos no $6^{\circ}$ dia de armazenamento. Esta cera, por apresentar elevada concentração de soluto $(30 \%)$, por ter causado elevação do teor de $\mathrm{CO}_{2}$ e redução do teor de $\mathrm{O}_{2}$ muito intensas no interior da fruta, resultou em efeitos indesejáveis, conforme preconizado por McGuire (1997), Baldwin et al. (1999) e Amarante et al. (2001).

A aplicação de ceras reduziu a perda de massa ao longo do armazenamento (Tabela 4). As frutas não tratadas apresentaram a maior perda de massa $(8,41 \%)$ após 6 dias, diferindo estatisticamente dos demais tratamentos, enquanto que as tratadas com Cleantex wax e Citrosol M mostraram as menores perdas. Em todos os tratamentos as perdas foram inferiores ao limite de $15 \%$ para goiabas em condições de consumo, segundo relatado por Manica et al. (2000). McGuire (1997) observou perdas de 9,2 e 13,3\% quando tratou goiabas Ruby com ceras e armazenou por sete dias a $12^{\circ} \mathrm{C}$.

Os valores da acidez total titulável (ATT) apresentaram variações durante o armazenamento, reduzindo-se de $0,62 \%$ de ácido cítrico 
no momento da colheita para valores entre 0,54 e $0,55 \%$ ao final do $6^{\circ}$ dia de conservação (Tabela 5). Estes resultados são semelhantes aos observados por Mercado-Silva et al. (1998) que também verificaram diminuição no teor de acidez durante armazenamento de goiabas a $25^{\circ} \mathrm{C}$.

TABELA 3 - Cor da polpa de goiabas Pedro Sato submetidas a aplicação de emulsões de cera de carnaúba e armazenadas a $25^{\circ} \mathrm{C}^{1}$.

\begin{tabular}{lccc}
\multicolumn{4}{c}{ de emulsões de cera de carnaúba e armazenadas a $25^{\circ} \mathrm{C}^{1}$} \\
\hline \multirow{2}{*}{ Tratamento } & \multicolumn{4}{c}{ Período de armazenamento (dias) } \\
\cline { 2 - 4 } & $\mathbf{2}$ & $\mathbf{4}$ & $\mathbf{6}$ \\
\hline Citrosol AK & ----------- Chroma & ------------ \\
& 35,31 & $43,25 \mathrm{a}$ & $43,96 \mathrm{a}$ \\
Fruit wax & 34,81 & $42,77 \mathrm{a}$ & $43,62 \mathrm{a}$ \\
Citrosol M & 35,65 & $43,45 \mathrm{a}$ & $44,48 \mathrm{a}$ \\
Meghwax ECF-100 & 34,73 & $40,15 \mathrm{~b}$ & $40,74 \mathrm{~b}$ \\
Controle & 35,62 & $41,84 \mathrm{ab}$ & $45,68 \mathrm{a}$ \\
Cleantex wax & 35,15 & $41,64 \mathrm{ab}$ & $43,90 \mathrm{a}$ \\
\hline Médias & 35,21 & 42,18 & 43,73 \\
Teste F (tratamento) & n.s. & $*$ & $* *$ \\
C. V. (\%) & 2,06 & 2,33 & 2,43 \\
\hline
\end{tabular}

${ }^{1}$ Médias seguidas da mesma letra na coluna não diferem pelo teste de Tukey a $5 \%$; **, * e n.s. = significativo a $\mathrm{P} \leq 0,01, \mathrm{P} \leq 0,05$ e não significativo, respectivamente.

${ }^{2}$ Chroma das frutas recém colhidas $=32,62$.

TABELA 4 - Perda de massa de goiabas Pedro Sato submetidas à aplicação de emulsões de cera de carnaúba e armazenadas a $25^{\circ} \mathrm{C}^{1}$.

\begin{tabular}{|c|c|c|c|}
\hline \multirow{2}{*}{ Tratamento } & \multicolumn{3}{|c|}{ Período de armazenamento (dias) } \\
\hline & 2 & 4 & 6 \\
\hline & & $--\%$ & \\
\hline Citrosol AK & $2,02 \mathrm{abc}$ & $4,15 \mathrm{~b}$ & $7,02 \mathrm{~b}$ \\
\hline Fruit wax & $2,23 \mathrm{ab}$ & $4,16 \mathrm{~b}$ & $5,99 \mathrm{bc}$ \\
\hline Citrosol M & $1,84 \mathrm{bc}$ & $3,34 \mathrm{c}$ & $5,65 \mathrm{c}$ \\
\hline Meghwax ECF-100 & $2,48 \mathrm{a}$ & $4,91 \mathrm{a}$ & $6,96 \mathrm{~b}$ \\
\hline Controle & $2,43 \mathrm{a}$ & $5,14 \mathrm{a}$ & $8,41 \mathrm{a}$ \\
\hline Cleantex wax & $1,76 \mathrm{c}$ & $3,73 \mathrm{bc}$ & $5,58 \mathrm{c}$ \\
\hline Médias & 2,13 & 4,24 & 6,60 \\
\hline Teste F (tratamento) & $* *$ & $* *$ & $* *$ \\
\hline C. V. $(\%)$ & 9,74 & 7,84 & 7,92 \\
\hline
\end{tabular}

${ }^{1}$ Médias seguidas da mesma letra na coluna não diferem pelo teste de Tukey a $5 \% ; * *=$ significativo a $\mathrm{P} \leq 0,01$.

TABELA 5 - Acidez total titulável de goiabas Pedro Sato submetidas à aplicação de emulsões de cera de carnaúba e armazenadas a $25^{\circ} \mathrm{C}^{1}$

\begin{tabular}{lccc}
\hline \multirow{2}{*}{ Tratamento } & \multicolumn{3}{c}{ Período de armazenamento (dias) } \\
\cline { 2 - 4 } & $\mathbf{2}$ & $\mathbf{4}$ & $\mathbf{6}$ \\
\hline & -----------0 & \% ácido cítrico & ------- \\
Citrosol AK & 0,607 & $0,625 \mathrm{a}$ & 0,543 \\
Fruit wax & 0,589 & $0,577 \mathrm{ab}$ & 0,539 \\
Citrosol M & 0,569 & $0,553 \mathrm{~b}$ & 0,521 \\
Meghwax ECF-100 & 0,598 & $0,561 \mathrm{ab}$ & 0,555 \\
Controle & 0,622 & $0,629 \mathrm{a}$ & 0,550 \\
Cleantex wax & 0,595 & $0,569 \mathrm{ab}$ & 0,547 \\
\hline Médias & 0,597 & 0,586 & 0,542 \\
Teste F (tratamento) & n.s. & $*$ & n.s. \\
C.V. (\%) & 6,32 & 5,43 & 5,95 \\
\hline
\end{tabular}

${ }^{1}$ Médias seguidas da mesma letra na coluna não diferem pelo teste de Tukey a $5 \%$; * e n.s = significativo a $\mathrm{P} \leq 0,05$ e não significativo, respectivamente.

${ }^{2}$ ATT das frutas recém colhidas: $0,624 \%$.

Houve redução no conteúdo de ácido ascórbico com o tempo de armazenamento (Tabela 6), de $64,32 \mathrm{mg} \cdot 100 \mathrm{~g}^{-1}$ de polpa, para valores entre 47,98 e 52,77 mg. $100 \mathrm{~g}^{-1}$ de polpa ao final de 6 dias de armazenamento. Yamashita \& Benassi (2000), trabalhando com goiabas Pedro Sato, ob- servaram valores médios de ácido ascórbico maiores que os encontrados neste experimento - em torno de $135,58 \mathrm{mg}^{100 \mathrm{~g}^{-1}}$ de polpa. Segundo Pereira (1996) e Mattheis \& Fellman (1999), vários fatores podem influenciar o teor de vitamina $\mathrm{C}$ para uma mesma variedade, tais como a época da colheita, o estádio de maturação e as condições climáticas durante o desenvolvimento das frutas.

O teor SST apresentou pequeno aumento após a colheita, passando de $8,56^{\circ}$ Brix no momento da colheita para valores entre $9,00 \mathrm{e}$ $10,31^{\circ}$ Brix ao final do experimento (Tabela 7). Estes resultados são diferentes dos observados por Yamashita \& Benassi (2000), que verificaram redução no teor de SST após a colheita de goiabas Pedro Sato. É possível que a degradação de polissacarídeos tenha contribuído para o aumento no teor de SST durante o armazenamento, pela liberação de hexoses (Awad, 1993), superando o consumo de açúcares na respiração. Além disso, a perda de massa, que foi de $6,6 \%$, em média, pode ter contribuído para concentrar os açúcares (Chitarra \& Chitarra, 1990; Tucker, 1993).

TABELA 6 - Teor de ácido ascórbico de goiabas Pedro Sato submetidas à aplicação de emulsões de cera de carnaúba e armazenadas a $25^{\circ} \mathrm{C}^{1}$.

\begin{tabular}{lccc}
\hline \multirow{2}{*}{ Tratamento } & \multicolumn{3}{c}{ Período de armazenamento (dias) ${ }^{2}$} \\
\cline { 2 - 4 } & $\mathbf{2}$ & $\mathbf{4}$ & $\mathbf{6}$ \\
\hline \multirow{2}{*}{ mitrosol AK } & \multicolumn{2}{c}{ me ácido ascórbico por } & $\mathbf{1 0 0 g}$ de polpa \\
Fruit wax & 60,13 & $57,97 \mathrm{~b}$ & $51,36 \mathrm{ab}$ \\
Citrosol M & 56,46 & $51,38 \mathrm{~b}$ & $50,70 \mathrm{ab}$ \\
Meghwax ECF-100 & 58,54 & $54,62 \mathrm{~b}$ & $52,77 \mathrm{ab}$ \\
Controle & 62,68 & $50,48 \mathrm{~b}$ & $47,98 \mathrm{~b}$ \\
Cleantex wax & 61,62 & $66,80 \mathrm{a}$ & $57,46 \mathrm{a}$ \\
Médias & 60,48 & $54,45 \mathrm{~b}$ & $52,38 \mathrm{ab}$ \\
Teste F (tratamento) & 59,98 & 55,95 & 52,11 \\
C.V. $(\%)$ & n.s. & $* *$ & $*$ \\
\hline
\end{tabular}

${ }^{1}$ Médias seguidas da mesma letra na coluna não diferem pelo teste de Tukey a $5 \% ; * * *$ e n.s. $=$ significativo a $\mathrm{P} \leq 0,01, \mathrm{P} \leq 0,05$ e não significativo, respectivamente.

${ }^{2}$ Teor de ácido ascórbico das frutas recém colhidas: $64,32 \mathrm{mg} / 100 \mathrm{~g}$.

TABELA 7 - Teor de sólidos solúveis totais de goiabas Pedro Sato submetidas à aplicação de emulsões de cera de carnaúba e armazenadas a $25^{\circ} \mathrm{C}^{1}$.

\begin{tabular}{|c|c|c|c|}
\hline \multirow{2}{*}{ Tratamento } & \multicolumn{3}{|c|}{ Período de armazenamento (dias) ${ }^{2}$} \\
\hline & 2 & 4 & 6 \\
\hline Citrosol AK & $8,15 \mathrm{c}$ & $\begin{array}{l}{ }^{\circ} \text { Brix -- } \\
9,00 \text { b }\end{array}$ & $9.00 \mathrm{~b}$ \\
\hline Fruit wax & $8,81 \mathrm{ab}$ & $9,13 \mathrm{ab}$ & $9,19 \mathrm{ab}$ \\
\hline Citrosol M & $8,69 \mathrm{bc}$ & $9,25 \mathrm{ab}$ & $9,00 \mathrm{~b}$ \\
\hline Meghwax ECF-100 & $9,19 \mathrm{ab}$ & $9,25 \mathrm{ab}$ & $9,69 \mathrm{ab}$ \\
\hline Controle & $9,38 \mathrm{a}$ & $9,94 \mathrm{ab}$ & $9,94 \mathrm{ab}$ \\
\hline Cleantex wax & $8,69 \mathrm{bc}$ & $10,13 \mathrm{a}$ & $10,31 \mathrm{a}$ \\
\hline Médias & 8,82 & 9,45 & 9,52 \\
\hline Teste F (tratamento) & $* *$ & $*$ & $* *$ \\
\hline C. V. $(\%)$ & 3,34 & 5,10 & 5,36 \\
\hline
\end{tabular}

${ }^{1}$ Médias seguidas da mesma letra na coluna não diferem pelo teste de Tukey a $5 \%$; ** e * significativo a $\mathrm{P} \leq 0,01$ e $\mathrm{P} \leq 0,05$.

${ }^{2} \mathrm{SST}$ das frutas recém colhidas: $8,56^{\circ} \mathrm{Brix}$.

A porcentagem de frutas com podridões no $6^{\circ}$ dia de armazenamento apresentou variação de 5 a $30 \%$ (Figura 1). As frutas do Controle apresentaram a maior percentagem de podridões $(30 \%)$, enquanto que as tratadas com Meghwax ECF-100 apresentaram a menor incidência $(5 \%)$. Nos demais tratamentos, a porcentagem de frutas com podridões variou de 10 a $20 \%$. Este fato, provavelmente, foi devido à atmosfera modificada promovida pela cera, que retardou o amadurecimento e a senescência das frutas, reduzindo a susceptibilidade dos tecidos à infecção por patógenos (Petracek et al., 1998; Durigan, 1999; Benato, 1999; Lana \& Finger, 2000). 
De maneira geral, a cera Meghwax ECF-100 foi a que mais contribuiu para retardar o amadurecimento das goiabas. As frutas tratadas com este produto mantiveram a coloração verde por mais tempo em relação às não tratadas. Esta cera proporcionou brilho e menor incidência de podridão. Por outro lado, estas goiabas apresentaram menor evolução da cor da polpa, além de discretos sabor e odor alcoólicos, possivelmente devido ao início do processo fermentativo (Figura 2). Essas alterações podem ser atribuídas à alta concentração de cera de carnaúba (30\%) contida no produto, uma vez que, a concentração de soluto é um dos fatores que interfere na taxa de permeabilidade da emulsão. Isso, provavelmente, ocasionou um aumento na concentração de $\mathrm{CO}_{2}$ e diminuição do nível de $\mathrm{O}_{2}$ no interior da fruta para níveis indesejáveis (Salunke \& Desai, 1984; McGuire, 1997; Baldwin et al., 1999).

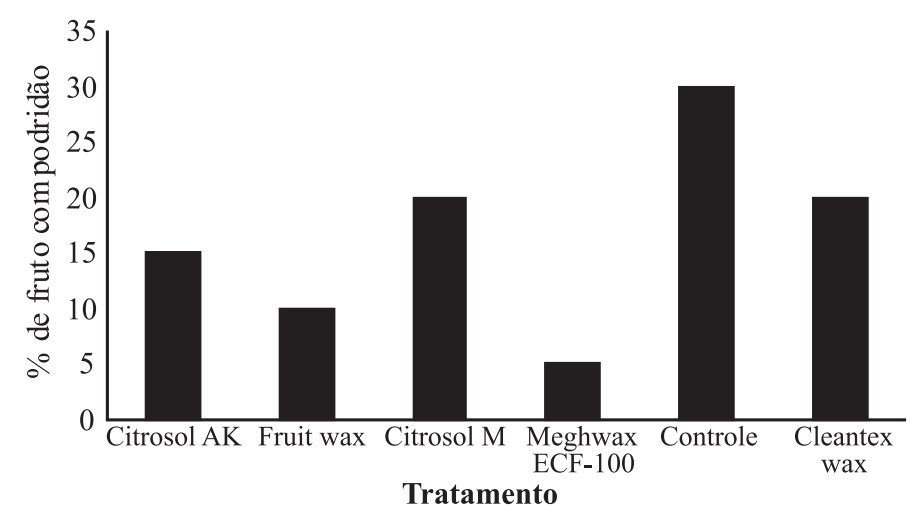

FIGURA 1 - Incidência de podridão em goiabas Pedro Sato no sexto dia após tratamento com emulsões de cera de carnaúba e armazenadas a $25^{\circ} \mathrm{C}$.

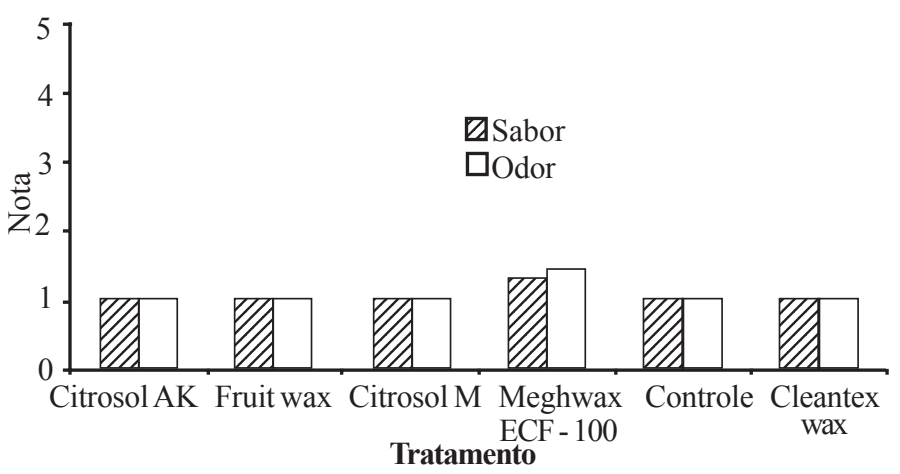

FIGURA 2 - Sabor e odor estranhos em goiabas Pedro Sato, no sexto dia após tratamento com emulsões de cera de carnaúba e armazenadas a $25^{\circ} \mathrm{C}$, de acordo com a seguinte escala de notas: $1=$ ausente, $2=$ leve, $3=$ moderado, $4=$ =intenso e $6=$ muito intenso.

\section{CONCLUSÕES}

1) A utilização de ceras à base de carnaúba é uma alternativa para ampliar o tempo de conservação de goiabas Pedro Sato em condição ambiente.

2) A aplicação de ceras de carnaúba em goiabas Pedro Sato retarda o amadurecimento, reduz a incidência de podridões e a perda de massa, além de conferir maior brilho às mesmas.

3) A cera Meghwax ECF-100 apresenta potencial para utilização em goiabas, porém há necessidade de ser avaliada em maior diluição.

\section{AGRADECIMENTO}

À empresa VAL FRUTAS pela doação das goiabas utilizadas no experimento.

\section{REFERÊNCIAS BIBLIOGRÁFICAS}

AMARANTE, C.; BANKS, N.H.; GANESH, S. Effects of coating concentration, ripening stage, water status and fruit temperature on pear susceptibility discoloration. Postharvest Biology and Technology, Wageningen, v.21, p.283-290, 2001.

AWAD, M. Fisiologia pós-colheita de frutos. São Paulo: Nobel, 1993. $114 \mathrm{p}$.

BALDWIN, E.A.; BURNS, J.K.; KAZOKAS, W.; BRECHT, J.K.; HAGENMAIER, R.D.; BENDER, R.J.; PESIS, E. Effect of two edible coating with different permeability characteristics on mango (Mangifera indica L.) ripening during storage. Postharvest Biology and Technology, Wageningen, v.17, p.215-226, 1999.

BENATO, E.A. Controle de doenças pós-colheita em frutas tropicais. Summa Phytopathologica, Jaboticabal, v.25, p.90-93, 1999.

BROWN, B.I.; WILLS, R.B.H. Post-harvest changes in guava fruit of different maturing. Scientia Horticulturae, Amsterdam, v.19, p.237243, 1983.

CARVALHO, C.R.L.; MANTOVANI, D.M.B.; CARVALHO, P.R.N.; MORAES, R.M.M. Análises químicas de alimentos. Campinas: ITAL, 1990. 121p. (Manual Técnico).

CHITARRA, M.I.F.; CHITARRA, A.B. Pós-colheita de frutos e hortaliças: fisiologia e manuseio. Lavras: ESAL/FAEPE, 1990. 320p.

DURIGAN, J.F. Uso da modificação da atmosfera no controle de doenças. Summa Phytopathologica, Jaboticabal, v.25, p.83-88, 1999.

GORRIS, L.G.M.; PEPPELENBOS, H.W. Modified atmosphere and vacuum packaging to extend the shelf life of respiring food products. HortTechnology, Alexandria, v.2, n.3, p.303-309, 1992.

HAGENMAIER,R.D.; BAKER, R.A. Wax microemulsions and emulsions as citrus coating. Journal of Agriculture Food Chemestry, Washington, v.42, p. 899-902, 1994.

JACOMINO, A.P. Conservação de goiabas 'Kumagai' em diferentes temperaturas e materiais de embalagem. 1999. 90f. Tese (Doutorado)Escola Superior de Agricultura "Luiz de Queiroz", Universidade de São Paulo, Piracicaba, 1990.

LANA, M.M.; FINGER, F.L. Atmosfera modificada e controlada: aplicação na conservação de produtos hortícolas. Brasília: EMBRAPA Hortaliças, 2000. 34p.

MANICA, I.; ICUMA, I.M.; JUNQUEIRA, N.T.V.; SALVADOR, J.O.; MOREIRA, A.; MALAVOLTA, E. Goiaba. Porto Alegre: Cinco Continetntes, 2000. 374p.

MATTHEIS, J.P.; FELLMAN, J.K. Preharvest factors influencing flavor of fresh fruit and vegetables. Postharvest Biology and Technology, Wageningen, v.15, p.227-232, 1999.

McGUIRE, R.G. Reporting of objective color measurements. HortScience, Alexandria, v.27, p.1254-1255, 1992.

McGUIRE, R.G. Market quality of guavas after hot-water quarantine treatment and application of carnauba wax coating. HortScience, Alexandria, v.32, p.271-274, 1997.

McGUIRRE R.G.; HALLMAN, G.J. Coating guavas with cellulose or carnauba-based emulsions interferes with postharvest ripening. Postharvest Biology and Tecnology, Wageningen, v.30, n.2, p.294295, 1995.

MERCADO-SILVA, E.; BENITO-BAUTISTA, P.; GARCIA-VELASCO, M.A. Fruit development, harvest index and ripening changes of guavas produced in central Mexico. Postharvest Biology and Technology, Wageningen, v.13, p.143-150, 1998.

NASCIMENTO, L.M. do; SANTOS, R.R. dos; RIBEIRO, I.J.A.; MARTINS, F.P.; YOTSUYANAGI, K.; COUTINHO, J.R. Caracterização físico-química dos frutos de 23 cultivares de goiabeiras (Psidium guajava L.) durante o processo de maturação. Revista Brasileira de Fruticultura, Cruz das Almas, v.13, p.25-34, 1991a.

NASCIMENTO, L.M. do; SANTOS, R.R. dos; RIBEIRO, I.J.A.; MARTINS, F.P.; YOTSUYANAGI, K.; COUTINHO, J.R. Caracterização físico-química dos frutos de 22 cultivares de goiabeiras ( Psidium guajava L.) durante o processo de maturação. Revista Brasileira 
de Fruticultura, Cruz das Almas, v.13, p.35-42, 1991 b.

PEREIRA, W.E. Desenvolvimento dos ramos e frutos de seis variedades de goiabeira (Psidium guajava L.) no período seco do ano. 1996. 48 f. Dissertação (M.S.) - Universidade Federal de Viçosa, Viçosa, 1996.

PETRACEK, P.D.; DOU, H.; PAO, S. The influence of applied waxes on postharvest physiological behavior and pitting of grapefruit. Postharvest Biology and Technology, Wageningen, v.14, p.99-106, 1998.

PIVETTA, K.F.L.; DURIGAN, J.F.; PEREIRA, F.M. Efeito de dois tipos de cera na conservação pós-colheita de frutos de goiabeira (Psidium guajava L.). Revista Brasileira de Fruticultura, Cruz das Almas, v.14,p.241-243, 1992.

SINGH, K.; CHAUHAN, K.S. Effect of certain postharvest treatments on storage life of cv. L-49 of guava. Haryana Journal of Horticultural Sciences, New Delhi, v. 11, n. 3/4, p. 163-167, 1982.

SINGH, R.V.; JOSHI, M.C.; RAM, H.B.; BISHT, N.S. Effect of wax coating and prepackaging on the storage behavior of guava cv. allahabad safeda. Indian Food Packer, New Delhi, v.38, n.6, p.80-85, 1984.

SALUNKE, D.K.; DESAI, B.B. Postharvest biotechnology of fruits. Boca Raton: CRC Press, 1984, v.2, p.39-46.

TAVARES, J.C. Efeitos da refrigeração, cera, fungicida e cálcio na conservação pós-colheita da goiaba 'Paluma' (Psidium guajava L.). 1993. 93f. Tese (Doutorado) - Faculdade de Ciências Agrárias e Veterinárias, Universidade Estadual Paulista "Júlio de Mesquita Filho", Jaboticabal, 1993.

TUCKER, G.A. Introduction. In: SEYMOUR, G.B.; TAYLOR, J.E.; TUCKER, G.A. Biochemistry of fruit ripening. London: Chapman \& Hall, 1993. Chapter 1, p.1-52.

YAMASHITA, F.; BENASSI, M.T. Influência da embalagem de atmosfera modificada e do tratamento com cálcio na cinética de degradação de ácido ascórbico e perda de massa em goiabas (Psidium guajava L.). Ciência e Tecnologia de Alimentos, Campinas, v.20, p.27-31, 2000. 